\title{
Obesity prevention and the Health promoting Schools framework: essential components and barriers to success
}

\author{
Rebecca Langford ${ }^{1 *}$, Christopher Bonell ${ }^{2}$, Hayley Jones ${ }^{3}$ and Rona Campbell ${ }^{1}$
}

\begin{abstract}
Background: Obesity is an important public health issue. Finding ways to increase physical activity and improve nutrition, particularly in children, is a clear priority. Our Cochrane review of the World Health Organization's Health Promoting Schools (HPS) framework found this approach improved students' physical activity and fitness, and increased fruit and vegetable intake. However, there was considerable heterogeneity in reported impacts. This paper synthesises process evaluation data from these studies to identify factors that might explain this variability.
\end{abstract}

Methods: We searched 20 health, education and social-science databases, and trials registries and relevant websites in 2011 and 2013. No language or date restrictions were applied. We included cluster randomised controlled trials. Participants were school students aged 4-18 years. Studies were included if they: took an HPS approach (targeting curriculum, environment and family/community); focused on physical activity and/or nutrition; and presented process evaluation data. A framework approach was used to facilitate thematic analysis and synthesis of process data.

Results: Twenty-six studies met the inclusion criteria. Most were conducted in America or Europe, with children aged 12 years or younger.

Although interventions were acceptable to students and teachers, fidelity varied considerably across trials. Involving families, while an intrinsic element of the HPS approach, was viewed as highly challenging. Several themes emerged regarding which elements of interventions were critical for success: tailoring programmes to individual schools' needs; aligning interventions with schools' core aims; working with teachers to develop programmes; and providing on-going training and support. An emphasis on academic subjects and lack of institutional support were barriers to implementation.

Conclusions: Stronger alliances between health and education appear essential to intervention success. Researchers must work with schools to develop and implement interventions, and to evaluate their impact on both health and educational outcomes as this may be a key determinant of scalability. If family engagement is attempted, better ways to achieve this must be developed and evaluated. Further evaluations of interventions to promote physical activity and nutrition during adolescence are needed. Finally, process evaluations must move beyond simple measures of acceptability/fidelity to include detailed contextual information to illuminate exactly what works, for whom, in what contexts and why.

Keywords: Children, Adolescents, Interventions, Schools, Physical activity, Healthy eating, Process evaluation, Health promoting Schools

\footnotetext{
* Correspondence: beki.langford@bristol.ac.uk

${ }^{1}$ DECIPHer, School of Social and Community Medicine, University of Bristol,

Canynge Hall, 39 Whatley Rd, Bristol BS8 2PS, UK

Full list of author information is available at the end of the article
} 


\section{Introduction}

Obesity is a pressing public health issue. In the past three decades rates of overweight and obesity have increased dramatically in most industrialised countries, with increases also observed in several low-income contexts [1]. This global epidemic is of particular concern for children and young people. Almost a third of children in America and a fifth in Europe are overweight or obese [2,3]. Childhood obesity strongly 'tracks' into adulthood [4], with implications for morbidity and premature mortality [5].

Obesity is a complex condition requiring equally complex solutions. The World Health Organization (WHO) suggests this requires action in multiple settings, using a variety of approaches and involving diverse stakeholders [6]:p16. A key element is complex, multi-component interventions implemented in schools targeting key determinants of obesity: namely, physical activity and nutrition [7].

One such approach is the WHO's Health Promoting Schools (HPS) framework. The HPS framework recognises the inherent, reciprocal link between health and education: healthy children achieve better educational outcomes which, in turn, are associated with better health later in life [8]. Inspired by the principles of the Ottawa charter and cognisant of the failure of health education alone to improve health outcomes, the HPS framework takes an eco-holistic approach to creating school environments conducive to health and healthy behaviours [9].

While definitions vary [8-14], HPS initiatives comprise: (1) health education promoted through the formal school curriculum; (2) changes to the school's physical and/or social environment; and (3) engagement with families and the wider community in recognition of the influence of these on children's health.

The HPS framework has proved popular in tackling obesity and other important public health issues such as cardio-vascular disease and Type II diabetes [11,15-17]. Half of the 67 trials included in our recent Cochrane review of the HPS framework targeted physical activity and/or nutrition [17]. Overall, we found intervention effects for improvements in students' levels of physical activity, physical fitness, and fruit and vegetable intake. We found no overall effect for reducing students' fat intake. The evidence for BMI and zBMI (standardised by age and gender) was equivocal. Surprisingly, given the underlying aim of the HPS framework, no study presented data on student academic attainment or attendance [17].

Within these studies we identified considerable heterogeneity in intervention effects. Given the complexity of these interventions and the variability between studies, it is important to consider why some were effective while others were not. Process evaluations can suggest explanations, helping to identify what works, for whom, in what contexts and why [18].

The aim of this paper was to synthesise process evaluation data presented in these studies to identify factors that helped or hindered implementation and/or success. Our findings have implications for the development of future trials and the implementation of programmes beyond the trial context.

\section{Methods}

\section{Inclusion criteria}

Full details of the methods can be found in the Cochrane review [17]. We included cluster randomised controlled trials (RCTs), with clusters at the level of school, district or other geographical area. Participants were students aged four to 18 years attending schools/colleges. As the HPS framework is not necessarily a term recognised in all countries, we did not require interventions to be explicitly based on the HPS framework. Rather, to be eligible interventions had to demonstrate active engagement in all three HPS domains, namely: curriculum, environment, and families and/or communities. Control schools offered no intervention or standard practice, or implemented an alternative intervention that included only one or two of the HPS criteria. For the purposes of this synthesis of process data, studies were included if they: took an HPS approach; focused on physical activity and/or nutrition; and presented process evaluation data.

\section{Search strategy}

We searched the following databases and trials registries using broad and inclusive search terms: ASSIA, Australian Education Index, British Education Index, BiblioMap, CAB Abstracts, Campbell Library, CENTRAL, CINAHL, Database of Educational Research, EMBASE, Education Resources Information Centre, Global Health Database, International Bibliography of Social Sciences, Index to Theses in Great Britain and Ireland, MEDLINE, PsycINFO, System for Information on Grey Literature in Europe, Social Science Citation Index, Sociological Abstracts, TRoPHI, Clinicaltrials.gov, Current Controlled Trials, and International Clinical Trials Registry Platform. We also searched relevant websites and reference lists of relevant articles. Searches were conducted in 2011 and 2013. No date or language restrictions were applied. One author performed an initial title screen, with a second screening a randomlyselected $10 \%$ of these for quality assurance (kappa score = 0.88 ). Thereafter, two reviewers independently screened abstracts and full texts to determine eligibility.

\section{Data extraction}

For each study, two reviewers independently extracted data pertaining to: study location, target age group, study 
duration, intervention content, outcome data and basic process data.

We undertook a thematic synthesis, adapting existing methods described by Thomas and Harden [19] to identify themes relating to programme implementation. More detailed descriptions of methods and quantitative and qualitative process findings were extracted verbatim from each study report by RL, that is extracting exactly the same words as the study author(s)' used. Data were also extracted from discussion sections of reports when this addressed implementation or reasons for intervention success or failure. These extracts were read and reread, an initial set of codes being developed and applied to the data. Some codes were identified a priori, focusing on aspects of process (acceptability, fidelity) while others arose inductively from the data (family involvement, barriers/facilitators). A Framework [20,21] approach was used to manage the data and assist analysis, whereby data from each study were summarised within a matrix under the following themes: intervention acceptability; implementation fidelity; family involvement; barriers to implementation; facilitators of implementation. This method allowed identification of similarities and differences between studies within themes.

\section{Results}

Our searches yielded 48,551 records (after deduplication), from which we identified 67 eligible studies (Figure 1). Of these, 34 focused on physical activity and/or nutrition. Twenty-six reported some process data and are the focus of this paper. Four studies focused solely on promoting physical activity, 11 on improving nutrition and 11 on physical activity and nutrition. Key characteristics of the interventions, including intervention activities

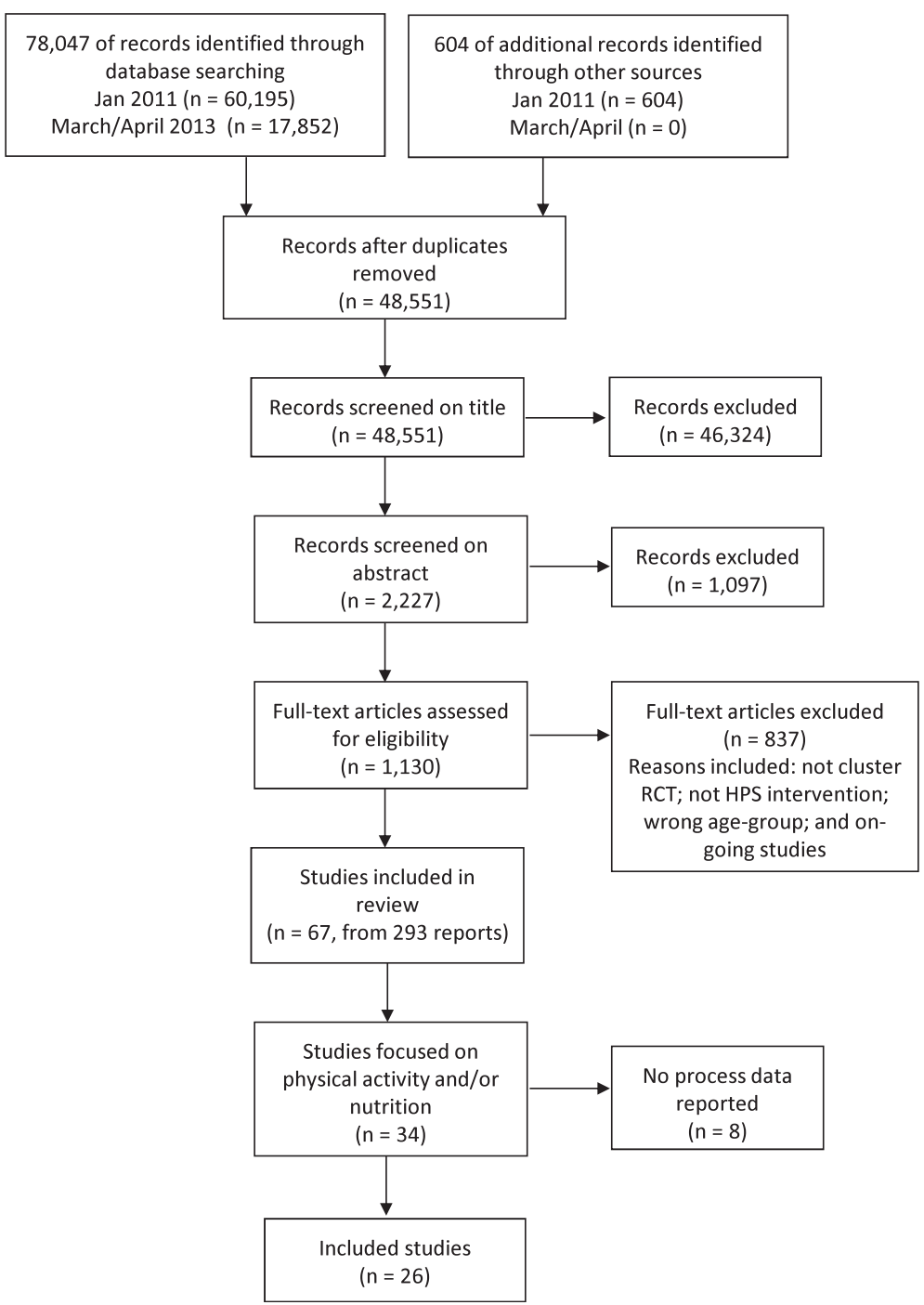

Figure 1 Flow chart of study selection process. 
carried out under the three HPS domains are presented in Table 1.

\section{Countries}

Thirteen studies were conducted in the USA $[24,25,27,28,30,33,43,46,47,51,54,55,58]$. A further 10 were implemented in Europe; two in the UK [23,52], two in Norway [22,49], one multi-country study (involving The Netherlands, Spain and Norway) [35] and one study each in Belgium [50], Finland [26], France [39], Germany [42] and Switzerland [38]. Three studies were conducted in Australia $[32,37,40]$.

\section{Age groups}

Of the 26 included studies, there were almost three times as many interventions conducted with younger children ( $\leq 12$ years) compared to older children (19 vs. 7 studies, respectively). Among the former, most targeted students aged between 8-12 years (11 studies), while five included younger children. Studies focusing on older students tended to be conducted with 12-14 year olds. Only one study was implemented in grade 9 (14-15 years).

\section{Quality of process data}

The methods used to collect process data are summarised in Table 1. The quality and extent of process data varied greatly. Some studies conducted extensive process evaluations, examining different elements of the intervention implementation. Most studies only focused on fidelity and acceptability using quantitative data, with diverse methods and scales bespoke for each intervention (e.g. questionnaires, log-books, structured observations). No study reported on the reliability or validity of these scales. Other studies provided extremely limited process data; it was often unclear how these were collected. Authors' conclusions about facilitators and barriers to implementation were included in some discussion sections but the evidence for these was generally unclear. However, these insights appeared to us useful and are included in our analysis. Few studies provided qualitative data. We summarise key themes below.

\section{Key themes}

\section{Acceptability}

Where reported, acceptability of the intervention to students, parents, and teaching or catering staff was generally high. Some studies provided quantitative assessments of acceptability. For example, teachers in the study by Bere et al. [22] all rated the intervention as 'good' or 'very good', while $70 \%$ of teachers and $90 \%$ of students participating in the KISS study reported they enjoyed the programme and wanted it to continue the following year [38]. Other studies reported acceptability in more general terms.
For example, Hoppu et al. merely stated that 'most of the feedback [on the intervention] was positive' [26]: p975. Resources (such as sports equipment [48], stickers to promote fruit and vegetable intake [25] or pedometers [41]) were often highly rated by teachers and students. Training and support provided to teachers were also highly appreciated $[44,52]$. One study conducted structured interviews with Physical Education (PE) teachers to assess the intervention's acceptability [48]. Some teachers were initially resistant to the new curriculum and implementation was lower in the early stages of the project. However, resistance lessened as they became familiar with the curriculum and could see the positive effect on student behaviour and activity levels.

\section{Fidelity}

Where reported, intervention fidelity varied. Some studies reported high levels of intervention fidelity [30,42,43,51,52]. In most studies, this was expressed as a percentage of intervention activities successfully implemented. For example, the $C A T C H$ trial reported $90 \%$ of food guidelines were met, $80 \%$ of PE activities were implemented and $88 \%$ of curriculum sessions were completed without modification [51]. Other studies reported much lower rates of implementation [22,23,27,34,46,54,58]. For example, one study [23] reported that despite high levels of acceptability, only $21 \%$ of intervention materials were implemented. Another [54] reported numerous problems with implementation, such as lack of volunteers or food preparation guidelines not being followed. Reynolds et al. [34] noted that taste-testing sessions were less likely to be undertaken because of the effort and disruption these caused. Importantly, they also noted that African-American and low socio-economic-status (SES) students were likely to receive lower doses of intervention activities, raising equity concerns. In the absence of additional contextual data, the authors were unable to explain this difference.

\section{Family involvement}

Despite being one of the three HPS domains, studies consistently identified engaging families as the most challenging and least successful intervention element. Almost all studies reporting on this indicated family engagement was low (typically only one-third to one-half of parents participating in any intervention activity) and authors frequently commented on the challenges of involving parents. The ICAPS trial noted parental attendance at meetings was poor (25-40\%), particularly in low SES areas [39]. Similarly, Eather and colleagues acknowledged 'parents are notoriously difficult to engage' and many students were not supported at home in completing their intervention's home-based activities [37]:p16. Two studies [36,41] identified language barriers in engaging non-English speaking 
Table 1 Characteristics of studies

\begin{tabular}{|c|c|c|c|c|}
\hline $\begin{array}{l}\text { Authors and } \\
\text { programme } \\
\text { name }\end{array}$ & Characteristics & HPS intervention elements & Summary of results & Process evaluation methods \\
\hline \multicolumn{5}{|c|}{ Nutrition interventions } \\
\hline $\begin{array}{l}\text { Bere et al. } \\
2006 \text { [22] }\end{array}$ & Country: & Curriculum: & \multirow{6}{*}{$\begin{array}{l}\text { No difference between intervention } \\
\text { and control groups for fruit and } \\
\text { vegetable intake. }\end{array}$} & \multirow{6}{*}{$\begin{array}{l}\text { Surveys to teachers, parents and students } \\
\text { to assess participation and acceptability } \\
\text { (assessed on likert scale). }\end{array}$} \\
\hline \multirow[t]{5}{*}{$\begin{array}{l}\text { Fruits and } \\
\text { Vegetables } \\
\text { Make the } \\
\text { Mark }\end{array}$} & Norway & $\begin{array}{l}\text { Curriculum was delivered in } \\
\text { Home Economics lesson over a } \\
\text { period of } 7 \text { months. Activities } \\
\text { included preparing fruit/veg-based } \\
\text { meals and snacks, taste testing, and } \\
\text { monitoring of fruit and vegetable } \\
\text { consumption over } 3 \text { days. }\end{array}$ & & \\
\hline & Target group: & Environment: & & \\
\hline & $11-12$ years & $\begin{array}{l}\text { Schools encouraged to participate } \\
\text { in the national fruit and vegetable } \\
\text { subscription programme. }\end{array}$ & & \\
\hline & Duration: & Family/community: & & \\
\hline & 6 months & Newsletters, parents meeting. & & \\
\hline $\begin{array}{l}\text { Evans et al. } \\
2013 \text { [23] }\end{array}$ & Country: & Curriculum: & \multirow{6}{*}{$\begin{array}{l}\text { No difference between intervention } \\
\text { and control groups for intake or } \\
\text { portions of fruit and vegetable } \\
\text { intake. }\end{array}$} & \multirow{6}{*}{$\begin{array}{l}\text { Questionnaires to teachers, parents and } \\
\text { students to assess implementation, } \\
\text { participation and acceptability (assessed on } \\
\text { likert scale). }\end{array}$} \\
\hline \multirow{5}{*}{$\begin{array}{l}\text { Project } \\
\text { Tomato }\end{array}$} & UK & $\begin{array}{l}\text { Teachers were provided with } \\
\text { twelve lesson plans. }\end{array}$ & & \\
\hline & Target group: & Environment: & & \\
\hline & $7-8$ years & $\begin{array}{l}\text { School health committee to } \\
\text { co-ordinate activities. }\end{array}$ & & \\
\hline & Duration: & Family/community: & & \\
\hline & 10 months & $\begin{array}{l}\text { Advice, newsletters and take- } \\
\text { home activity bags. }\end{array}$ & & \\
\hline \multirow{2}{*}{$\begin{array}{l}\text { Foster et al. } \\
2008 \text { [24] }\end{array}$} & Country: & Curriculum: & \multirow{7}{*}{$\begin{array}{l}\text { Incidence of overweight was } \\
\text { significantly lower for intervention } \\
\text { than control students ( } 7.5 \% \text { vs. } \\
14.9 \% \text {, adjusted odds ratio: } 0.67 \text {, } \\
95 \% \text { Cl } 0.47 \text { to } 0.96 \text { ). }\end{array}$} & \multirow{7}{*}{$\begin{array}{l}\text { Very minimal data provided on staff } \\
\text { training and hours of nutrition } \\
\text { education. No details on methods } \\
\text { provided. }\end{array}$} \\
\hline & USA & 50 hours of food and nutrition & & \\
\hline \multirow{5}{*}{$\begin{array}{l}\text { School } \\
\text { Nutrition } \\
\text { Policy } \\
\text { Initiative }\end{array}$} & & $\begin{array}{l}\text { education provided per year. The } \\
\text { curriculum was integrated into } \\
\text { various classroom subjects. }\end{array}$ & & \\
\hline & Target group: & Environment: & & \\
\hline & $9-12$ years & $\begin{array}{l}\text { Nutrition Advisory Group set up. } \\
\text { Changes made to food sold in } \\
\text { schools to ensure they met } \\
\text { nutritional standards. Other } \\
\text { activities included: limiting use of } \\
\text { food as a reward, promoting } \\
\text { active recess and providing } \\
\text { healthy breakfasts. }\end{array}$ & & \\
\hline & Duration: & Family/community: & & \\
\hline & 2 years & $\begin{array}{l}\text { Report card nights, parent } \\
\text { education meetings, and weekly } \\
\text { nutrition workshops. }\end{array}$ & & \\
\hline \multirow{2}{*}{$\begin{array}{l}\text { Hoffman } \\
\text { et al. } 2010 \\
\text { [25] }\end{array}$} & Country: & Curriculum: & \multirow{5}{*}{$\begin{array}{l}\text { By end of year } 2 \text {, intervention group } \\
\text { consumed more fruit (but not } \\
\text { vegetables) than the control group } \\
\text { ( } 34 \mathrm{~g} \text { vs. } 23 \mathrm{~g}, \mathrm{p}<0.001 \text { ). }\end{array}$} & \multirow{5}{*}{$\begin{array}{l}\text { Questionnaires to teachers, lunch aides } \\
\text { and students to assess acceptability } \\
\text { using 6-point likert scale. Unannounced } \\
\text { fidelity checks and observations of lunchtime } \\
\text { components. Log book of public service } \\
\text { announcements kept. }\end{array}$} \\
\hline & USA & $\begin{array}{l}\text { The classroom component } \\
\text { included the 5-A-Day Adventures }\end{array}$ & & \\
\hline \multirow{3}{*}{$\begin{array}{l}\text { Athletes in } \\
\text { Service, Fruit } \\
\text { and } \\
\text { Vegetable } \\
\text { Promotion } \\
\text { Program }\end{array}$} & & CD-ROM. & & \\
\hline & Target group: & Environment: & & \\
\hline & $5-7$ years & $\begin{array}{l}\text { Loudspeaker announcements } \\
\text { and posters to promote fruit/ } \\
\text { veg. Lunch aides praised } \\
\text { children eating fruit and } \\
\text { vegetables and offered stickers. }\end{array}$ & & \\
\hline
\end{tabular}


Table 1 Characteristics of studies (Continued)

\begin{tabular}{|c|c|c|c|c|}
\hline \multirow{8}{*}{$\begin{array}{l}\text { Hoppu et al. } \\
2010[26] \\
\text { (no name) }\end{array}$} & Duration: & Family/community & & \\
\hline & 2.5 years & $\begin{array}{l}\text { Family homework assignments. } \\
\text { Parents involved in creating a } \\
\text { school cookbook. }\end{array}$ & & \\
\hline & Country: & Curriculum: & \multirow{6}{*}{$\begin{array}{l}\text { No difference between intervention } \\
\text { and control group for fruit/veg } \\
\text { consumption. }\end{array}$} & \multirow{6}{*}{$\begin{array}{l}\text { Teachers, catering staff and students } \\
\text { asked to give their opinions on the } \\
\text { intervention (no further details provided } \\
\text { on methods or questions asked). } \\
\text { Teachers reported use of intervention } \\
\text { materials using on-line system. }\end{array}$} \\
\hline & Finland & $\begin{array}{l}\text { Nutrition education was } \\
\text { implemented by teachers during } \\
\text { regular lessons. }\end{array}$ & & \\
\hline & Target group: & Environment: & & \\
\hline & 13-14 years & $\begin{array}{l}\text { Sugary snacks restricted and } \\
\text { healthy alternatives encouraged. } \\
\text { Drama workshops held. }\end{array}$ & & \\
\hline & Duration: & Family/community: & & \\
\hline & 8 months & $\begin{array}{l}\text { Parent information meeting. } \\
\text { Healthy eating magazine. }\end{array}$ & & \\
\hline \multirow{6}{*}{$\begin{array}{l}\text { Lytle et al. } \\
2004 \text { [27] } \\
\text { TEENS }\end{array}$} & Country: & Curriculum: & \multirow{6}{*}{$\begin{array}{l}\text { No significant differences between } \\
\text { intervention and control groups for } \\
\text { fruit and vegetable intake. }\end{array}$} & \multirow{6}{*}{$\begin{array}{l}\text { Lesson checklists and observations of } \\
\text { classroom sessions. Teacher and student } \\
\text { evaluations of curriculum acceptability } \\
\text { assed via likert scale. Documentation of } \\
\text { family participation via number of } \\
\text { behavioural coupons returned and family } \\
\text { homework assignments completed. Visits } \\
\text { to food service teams. Logs of school } \\
\text { nutrition advisory councils. }\end{array}$} \\
\hline & USA & $\begin{array}{l}\text { Ten nutrition education lessons } \\
\text { were implemented in both grade } \\
7 \text { and } 8 . \text { These sessions involved } \\
\text { self-monitoring, goal setting, } \\
\text { hands-on snack preparation, and } \\
\text { skill development. }\end{array}$ & & \\
\hline & Target group: & Environment: & & \\
\hline & 12-14 years & $\begin{array}{l}\text { Changes made to school food } \\
\text { service to improve nutritional } \\
\text { quality of food. School Nutrition } \\
\text { Advisory Councils created. }\end{array}$ & & \\
\hline & Duration: & Family/community: & & \\
\hline & 2 years & $\begin{array}{l}\text { Newsletters and behavioural } \\
\text { coupons. }\end{array}$ & & \\
\hline \multirow{6}{*}{$\begin{array}{l}\text { Nicklas et al. } \\
1998[28,29]\end{array}$} & Country: & Curriculum: & \multirow{6}{*}{$\begin{array}{l}\text { No significant differences between } \\
\text { intervention and control groups for } \\
\text { fruit and vegetable intake. }\end{array}$} & \multirow{6}{*}{$\begin{array}{l}\text { Observations and evaluation forms for } \\
\text { training workshops. Logbooks document } \\
\text { family involvement via newsletters/ } \\
\text { calendar distribution and attendance at } \\
\text { parent-teacher meetings. Menu documen- } \\
\text { tation, salad bar assessments and food } \\
\text { use surveys. Monthly rates of participation } \\
\text { in school lunches recorded. }\end{array}$} \\
\hline & USA & $\begin{array}{l}\text { Five (55 minute) themed } \\
\text { workshops provided students } \\
\text { with learning opportunities to } \\
\text { develop knowledge, positive } \\
\text { attitudes and skills necessary to } \\
\text { increase fruit and vegetable } \\
\text { consumption. }\end{array}$ & & \\
\hline & Target group: & Environment: & & \\
\hline & 14-15 years & $\begin{array}{l}\text { School-wide media marketing } \\
\text { campaign was implemented } \\
\text { including taste testing, posters, } \\
\text { public service announcements } \\
\text { and student contests. School } \\
\text { meals modified to increase fruit } \\
\text { and veg provision. Staff training. }\end{array}$ & & \\
\hline & Duration: & Family/community: & & \\
\hline & 3 years & $\begin{array}{l}\text { Parents' brochures, newsletters } \\
\text { and a seasonal food calendar. } \\
\text { Events held at Parent-Teacher } \\
\text { Organization meetings. }\end{array}$ & & \\
\hline $\begin{array}{l}\text { Perry et al. } \\
1998[30,31] \\
5 \text { A DAY } \\
\text { Power Plus }\end{array}$ & $\begin{array}{l}\text { Country: } \\
\text { USA }\end{array}$ & $\begin{array}{l}\text { Sixteen } 40-45 \text { minutes classroom } \\
\text { sessions were implemented twice } \\
\text { a week for } 8 \text { weeks. Sessions }\end{array}$ & $\begin{array}{l}\text { Intervention students consumed } \\
\text { more servings of fruit (per } 100 \text { kcals, } \\
\text { difference } 0.41, P=0.02 \text { ) and } \\
\text { combined fruit and vegetable } \\
\text { servings (per } 100 \text { kcals, difference }\end{array}$ & $\begin{array}{l}\text { Training participation rates and feedback } \\
\text { from participants. Observations of } \\
\text { classroom activities and lunchtimes. }\end{array}$ \\
\hline
\end{tabular}


Table 1 Characteristics of studies (Continued)

\begin{tabular}{|c|c|c|c|c|}
\hline & & $\begin{array}{l}\text { included skills-building, problem- } \\
\text { solving and taste-testing. }\end{array}$ & $\begin{array}{l}0.36, P=0.02 \text { ) and less fat (as } \% \text { of } \\
\text { total kcal, difference }-1.81, P=0.02 \text { ). }\end{array}$ & \\
\hline & Target group: & Environment: & & \\
\hline & 9-11 years & $\begin{array}{l}\text { Changes made to school food } \\
\text { provision. Catering staff training. } \\
\text { Students were rewarded for } \\
\text { eating fruits and vegetables } \\
\text { during lunch. }\end{array}$ & & \\
\hline & Duration: & Family/community: & & \\
\hline & 6 months & $\begin{array}{l}\text { Home information/activity packs } \\
\text { were sent home. }\end{array}$ & & \\
\hline \multirow{6}{*}{$\begin{array}{l}\text { Radcliffe } \\
\text { et al. } 2005 \\
\text { [32] } \\
\text { (no name) }\end{array}$} & Country: & Curriculum: & \multirow{6}{*}{$\begin{array}{l}\text { No significant difference between } \\
\text { intervention and control groups for } \\
\% \text { students skipping breakfast. }\end{array}$} & \multirow{6}{*}{$\begin{array}{l}\text { No details on methods but table provided } \\
\text { number of schools implementing each } \\
\text { intervention component. }\end{array}$} \\
\hline & Australia & $\begin{array}{l}\text { Variety of changes to the } \\
\text { curriculum including: classes } \\
\text { focusing on health, nutrition and } \\
\text { breakfast; a unit on body image } \\
\text { and healthy eating; breakfast } \\
\text { information provided to teachers; } \\
\text { development of breakfast recipe } \\
\text { books and trailing of recipes etc. }\end{array}$ & & \\
\hline & Target group: & Environment: & & \\
\hline & 12-13 years & $\begin{array}{l}\text { Working group developed action } \\
\text { plans. Variety of activities } \\
\text { including: events to promote } \\
\text { breakfast; designating a breakfast } \\
\text { eating area; change to timetable } \\
\text { to enable earlier morning snack } \\
\text { times; trialling breakfast tuck } \\
\text { shops; improving nutritional } \\
\text { quality of breakfast foods sold at } \\
\text { the tuckshop. }\end{array}$ & & \\
\hline & Duration: & Family/community: & & \\
\hline & 11 months & $\begin{array}{l}\text { Variety of activities including: } \\
\text { newsletter; parent education } \\
\text { forums; involving parents in } \\
\text { classroom activities and special } \\
\text { events etc. }\end{array}$ & & \\
\hline \multirow{2}{*}{$\begin{array}{l}\text { Reynolds } \\
\text { et al. } 2000 \\
{[33,34]}\end{array}$} & Country: & Curriculum: & \multirow{7}{*}{$\begin{array}{l}\text { Intervention children consumed } \\
\text { significantly more fruit and } \\
\text { vegetables than the control group } \\
\text { (3.96 servings vs. } 2.28, P<0.0001 \text { ). }\end{array}$} & \multirow{7}{*}{$\begin{array}{l}\text { Questionnaire to curriculum } \\
\text { co-ordinators to assess acceptability } \\
\text { (on 5-point likert scale). Classroom } \\
\text { observations and checklists. Cafeteria } \\
\text { observations. Interviews with food } \\
\text { service managers. Assessments of } \\
\text { family involvement through checklists, } \\
\text { assessments of homework completion } \\
\text { and telephone parent surveys. }\end{array}$} \\
\hline & USA & $\begin{array}{l}\text { Nutrition curriculum (14 lessons) d } \\
\text { included modelling, self- }\end{array}$ & & \\
\hline \multirow[t]{5}{*}{ High 5} & & $\begin{array}{l}\text { monitoring, problem-solving, } \\
\text { reinforcement, taste testing and } \\
\text { other methods. }\end{array}$ & & \\
\hline & Target group: & Environment: & & \\
\hline & 9-10 years & $\begin{array}{l}\text { Food service managers training. } \\
\text { Cafeteria was rated on a monthly } \\
\text { basis and given } 2,3 \text { or } 4 \text { stars } \\
\text { based on their completion of } 10 \\
\text { intervention activities. }\end{array}$ & & \\
\hline & Duration: & Family/community: & & \\
\hline & 1 year & $\begin{array}{l}\text { Parents' 'kick-off' night. Family } \\
\text { homework assignments. }\end{array}$ & & \\
\hline \multirow{2}{*}{$\begin{array}{l}\text { Te Velde } \\
\text { et al. } 2008 \\
{[35,36]} \\
\text { Pro Children } \\
\text { Study }\end{array}$} & Country: & Curriculum: & \multirow{2}{*}{$\begin{array}{l}\text { At first follow-up intervention } \\
\text { children reported greater intake of } \\
\text { fruit and vegetables compared to } \\
\text { controls. However, by second } \\
\text { follow-up (one year later) a significant } \\
\text { effect was only seen in one country }\end{array}$} & \multirow{2}{*}{$\begin{array}{l}\text { Teacher questionnaire used to assess } \\
\text { fidelity (composite score created } \\
\text { ranging from 0-16). Student survey } \\
\text { to assess acceptability on 3-point } \\
\text { likert scale for different intervention } \\
\text { elements. Assessments of family } \\
\text { involvement via completion of }\end{array}$} \\
\hline & $\begin{array}{l}\text { Netherlands, } \\
\text { Norway, Spain }\end{array}$ & $\begin{array}{l}16 \text { worksheets aimed at } \\
\text { increasing knowledge, awareness } \\
\text { and skills. Included taste testing } \\
\text { activities and computerised } \\
\text { tailored feedback. }\end{array}$ & & \\
\hline
\end{tabular}


Table 1 Characteristics of studies (Continued)

\begin{tabular}{ll}
\hline Target group: & Environment: \\
$10-12$ years & $\begin{array}{l}\text { Free fruit/veg provided, Changes } \\
\text { to school food provision to } \\
\text { increase amount of fruit/veg } \\
\text { available. }\end{array}$ \\
& $\begin{array}{l}\text { Family/community: } \\
\text { Duration: }\end{array}$ \\
$\begin{array}{l}\text { Family homework assignments, } \\
\text { newsletters and a parent version } \\
\text { of the web-based computer- } \\
\text { tailored tool. }\end{array}$
\end{tabular}

\section{Physical activity interventions}

\begin{tabular}{|c|c|c|}
\hline & Country: & Home activity programme \\
\hline & Australia & comprised of 20 minutes physical \\
\hline Fit-4-Fun & Target group: & weeks. Work booklets, information \\
\hline & $10-12$ years & $\begin{array}{l}\text { and fitness challenges sent to } \\
\text { parents. }\end{array}$ \\
\hline & Duration: & \\
\hline & 8 weeks & \\
\hline Kriemler & Country: & Curriculum: \\
\hline $\begin{array}{l}\text { et al. } 2010 \\
\text { [38] }\end{array}$ & Switzerland & $\begin{array}{l}\text { Two additional PE lessons a week } \\
\text { were implemented by specialist }\end{array}$ \\
\hline KISS & & PE teachers. \\
\hline & Target group: & Environment: \\
\hline & $\begin{array}{l}6-7,10-11 \\
\text { years }\end{array}$ & $\begin{array}{l}\text { Several short activity breaks ( } 2-5 \\
\text { minutes) were introduced during } \\
\text { academic lesson every day. }\end{array}$ \\
\hline & Duration: & Family/community: \\
\hline & 11 months & $\begin{array}{l}\text { Flyers on health topics were sent } \\
\text { to parents }\end{array}$ \\
\hline Simon et al. & Country: & Curriculum: \\
\hline 2006 [39] & France & Curriculum focused on physical \\
\hline ICAPS & & $\begin{array}{l}\text { activity and sedentary behaviours. } \\
\text { Aimed to transmit knowledge } \\
\text { and skills about physical activity. }\end{array}$ \\
\hline & Target group: & Environment: \\
\hline & $11-12$ years & $\begin{array}{l}\text { Increased opportunities for } \\
\text { physical activity offered at breaks, } \\
\text { at lunchtimes and after school. }\end{array}$ \\
\hline & Duration: & Family/community: \\
\hline & 4 years & $\begin{array}{l}\text { Parents and teacher meetings. } \\
\text { Policy makers of local } \\
\text { communities were requested to } \\
\text { provide a supportive environment } \\
\text { that promote physical activity. For } \\
\text { example, free/low-cost entry to } \\
\text { sports facilities. }\end{array}$ \\
\hline Wen et al. & Country: & Curriculum: \\
\hline $2008[40,41]$ & Australia & Home to school mapping \\
\hline (no name) & & $\begin{array}{l}\text { exercise' used to help students } \\
\text { plan their active journey to high } \\
\text { school next year. Some schools } \\
\text { also used pedometers and an } \\
\text { associated classroom program. }\end{array}$ \\
\hline
\end{tabular}

(Norway, 91.5 additional grams per day, $95 \% \mathrm{Cl} 49.8$ to $133.2, \mathrm{p}=0.04)$.

Significant improvements in intervention group found for: fitness (adjusted mean difference, 1.14 levels, $p<0.001)$, BMI (mean, -0.96 $\mathrm{kg} / \mathrm{m} 2, \mathrm{p}<0.001) \mathrm{zBMl} z$-score mean $-0.47 z$-scores, $p<0.001$ ), flexibility (sit and reach mean, $1.52 \mathrm{~cm}, \mathrm{p}=$ $0.0013)$, muscular fitness (sit-ups) (mean 0.62 stages, $p=0.003$ ) and physical activity (mean, 3253 steps/ day, $p<0.001$ ).

Intervention children showed improvements in skinfold thickness $(-0.12,95 \% \mathrm{Cl}-0.21$ to -0.03$)$, fitness $(0.17,95 \% \mathrm{Cl} 0.01$ to 0.32$)$, school MVPA $(1.19,95 \% \mathrm{Cl} 0.78$ to 1.6) and all-day MVPA $(0.44,95 \% \mathrm{Cl}$ 0.05 to 0.82 ) and total physical activity in school $(0.92,95 \% \mathrm{Cl} 0.35$ to 1.5$)$.

Intervention students had a lower increase in BMI $(p<0.01)$ and age/ gender-adjusted BMI $(p<0.02)$. They also had increased participation in supervised physical activity $(p<0.001)$, a decrease in TV/video viewing $(p<0.01)$ and an increase in highdensity cholesterol concentrations $(p<0.001)$. family homework assignments, use of intervention's computer programme, and receipt of newsletters.
Questionnaires to teachers and students to assess participation and satisfaction on six-point likert scale.
Questionnaires to teachers and students to assess acceptability on 6-point likert scale.
No difference between intervention Semi-structured qualitative and control students reporting walking to school.
Quantitative documentation of number of activities provided and individual attendance at sessions. Number of school hours devoted to curriculum and school debates. Actions initiated by or in collaboration with outside partners were recorded. interviews with principals and/or teaching co-ordinators to assess fidelity and acceptability. 
Table 1 Characteristics of studies (Continued)

\begin{tabular}{ll}
\hline Target group: & Environment: \\
9-11 years & A consultation group comprised \\
& of teachers, parents and officers \\
& from local councils set up to \\
& encourage active commuting. \\
& Banners provided for schools. \\
& Walk Safely to School Day \\
& activities held. \\
& Family/community: \\
& Information on active travel \\
& provided to parent. Parent events \\
& and walks. Newsletters. Local \\
& councils reviewed safety and \\
& walkability of nearby participating \\
& schools and worked to make \\
& improvements.
\end{tabular}

Physical Activity + Nutrition Interventions

\section{Brandstetter Country: Curriculum: \\ et al. 2012 \\ [42] \\ Germany \\ URMEL ICE \\ Caballero \\ Country: Curriculum: \\ Target group: Environment: \\ 7-8 years Two short blocks of physical activity exercises (each 5-7 minutes) were implemented every day. Teachers training. \\ Duration: Family/community: \\ 9 months Family homework assignments and training and information materials.}

et al. 2003

[43-45]

Pathways physical activity and nutrition. In
Classroom curriculum designed to $3 r d$ and 4th grades, two 45-minute lessons delivered for 12 weeks. In 5 th grade this decreased to 8 weeks.

\section{Target group: Environment:}

8-9 years

School food service guidelines issues to decrease fat content of meals. Minimum of three 30-minute sessions of MVPA per week. Exercise breaks to promote physical activity in the classroom. Teacher training.

Duration:

\section{Family/community:}

3 years Family action packs. Family events, included cooking demonstrations.

\section{Crespo et al. Country: Curriculum:}

2012 [46]

Aventuras para Niños
SPARK physical activity curriculum implemented.

\section{Target group: Environment:}

5-8 years
Improvements were made to school playgrounds and salad bars. Physical activity equipment
No effect found on BMl, waist circumference and skin-fold thickness after adjustment for time lag between baseline and follow-up.

No difference between intervention and control for BMI or other anthropometric measures or physical activity levels. Intervention students reported lower total daily energy intake (1892 vs. $2157 \mathrm{kcal} / \mathrm{d}$ $\mathrm{p}=0.003)$ and percentage of energy from fat (31.1\% vs. $33.6 \%, p=0.001)$ than control students.

No difference between intervention and control groups for BMI.
Questionnaire to teachers to assess fidelity (teachers asked to indicate which teaching units has been used in class).
Quantitative assessments of the four main components of the intervention, including attendance log for training sessions and family events, PE calendars, kitchen visits and parent/student evaluation forms.
Direct observations and audit tools used to assess fidelity of intervention components (no further details provided). 
Table 1 Characteristics of studies (Continued)

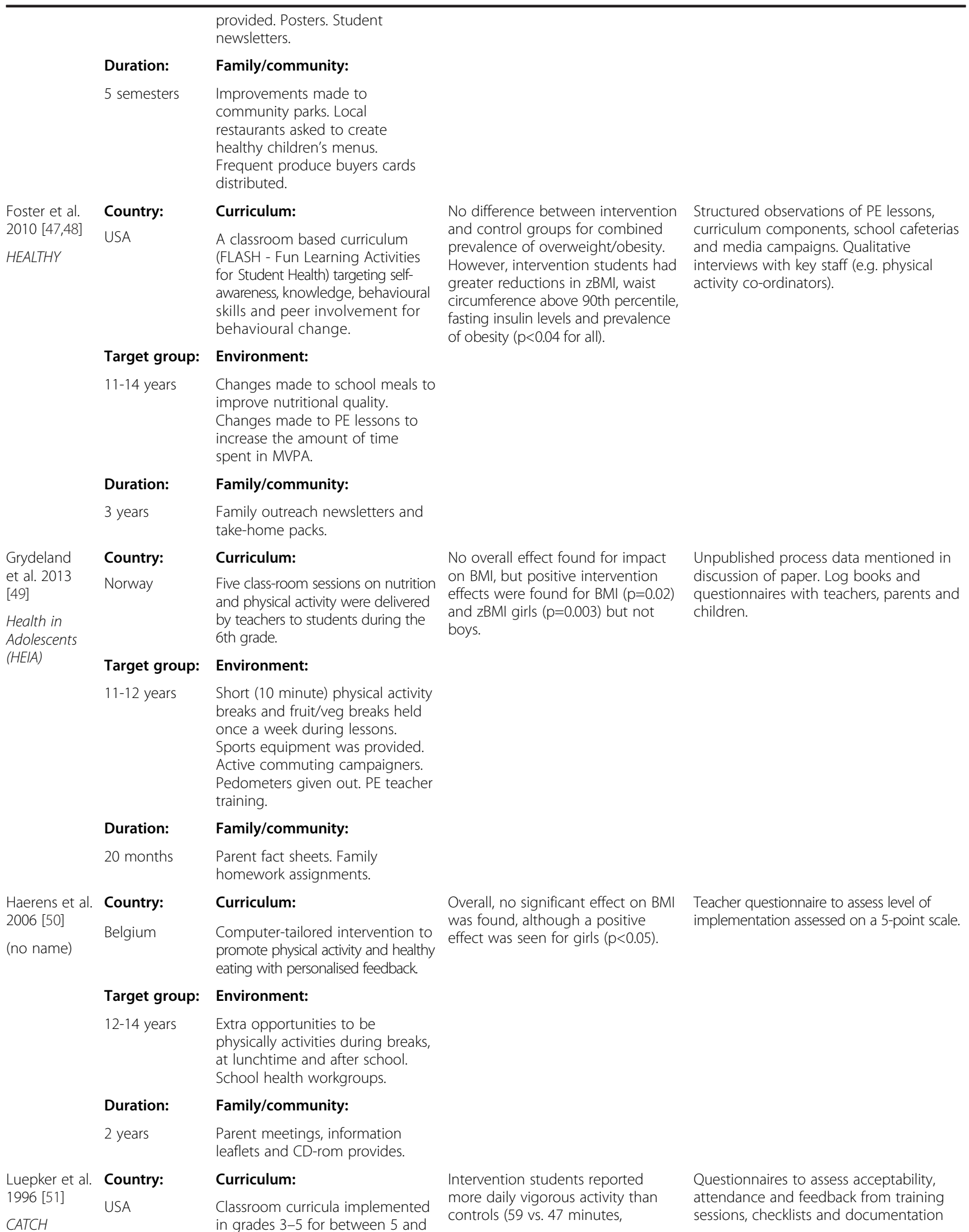


Table 1 Characteristics of studies (Continued)

\begin{tabular}{|c|c|c|c|c|}
\hline & & $\begin{array}{l}12 \text { weeks (depending on grade). } \\
\text { Each lesson was } 30-40 \text { minutes. } \\
\text { The curricula targeted } \\
\text { psychosocial factors and skills } \\
\text { development. }\end{array}$ & \multirow[t]{5}{*}{$\begin{array}{l}p<0.003) \text { and greater reductions in } \\
\text { daily energy intake from fat }(2.4 \% \\
\text { vs. } 0.4 \% \text { reductions, } p<0.001) \text {. }\end{array}$} & \multirow[t]{5}{*}{$\begin{array}{l}\text { logs for intervention activities, structured } \\
\text { observations of intervention activities. }\end{array}$} \\
\hline & Target group: & Environment: & & \\
\hline & 8-9 years & $\begin{array}{l}\text { Changes made to school meals to } \\
\text { improve nutritional content. } \\
\text { Catering staff training. Changes to } \\
\text { PE lessons to increase time spent } \\
\text { in MVPA. Teacher training. }\end{array}$ & & \\
\hline & Duration: & Family/community: & & \\
\hline & 3 years & $\begin{array}{l}\text { Activity packs were sent home to } \\
\text { be completed by students and } \\
\text { parents together. Family fun nights. }\end{array}$ & & \\
\hline \multirow{6}{*}{$\begin{array}{l}\text { Sahota et al. } \\
2001[52,53] \\
\text { APPLES }\end{array}$} & Country: & Curriculum: & \multirow{6}{*}{$\begin{array}{l}\text { No difference between intervention } \\
\text { and control group for overweight/ } \\
\text { obesity. Intervention children } \\
\text { consumed more vegetables than } \\
\text { control group (weighted mean } \\
\text { difference: } 0.3,95 \% \mathrm{Cl} 0.2 \text { to } 0.4 \text { ). }\end{array}$} & \multirow{6}{*}{$\begin{array}{l}\text { Teacher surveys (no further details } \\
\text { provided). Meals monitored by collection } \\
\text { of monthly menus, observations and } \\
\text { discussions with staff. }\end{array}$} \\
\hline & UK & $\begin{array}{l}\text { Nutrition education incorporated } \\
\text { into the curriculum, healthy } \\
\text { eating lessons delivered by the } \\
\text { project dietician and 'Fit is Fun' } \\
\text { programme incorporated into } \\
\text { physical education lessons. }\end{array}$ & & \\
\hline & Target group: & Environment: & & \\
\hline & 9-11 years & $\begin{array}{l}\text { Teacher training, modification of } \\
\text { school meals and the development } \\
\text { of school action plans designed to } \\
\text { promote healthy eating and } \\
\text { physical activity. }\end{array}$ & & \\
\hline & Duration: & Family/community: & & \\
\hline & 10 months & $\begin{array}{l}\text { Consultation with parents about } \\
\text { what the intervention should } \\
\text { include. Parents were invited to } \\
\text { help run sessions. Information on } \\
\text { intervention sent out to parents. }\end{array}$ & & \\
\hline $\begin{array}{l}\text { Sallis et al. } \\
2003[54]\end{array}$ & Country: & Curriculum: & \multirow{6}{*}{$\begin{array}{l}\text { Total physical activity levels } \\
\text { improved in the intervention group } \\
\text { compared to controls ( } d=0.93 \text {, } \\
p<0.009 \text { ). Subgroup analyses revealed } \\
\text { the intervention to only be effective } \\
\text { for boys. BMI was reduced in boys in } \\
\text { the intervention group ( } d-0.83 \text {, } \\
P=0.04 \text { ). No effect seen for total fat or } \\
\text { saturated fat. }\end{array}$} & \multirow{6}{*}{$\begin{array}{l}\text { Discussion section of paper describes } \\
\text { problems encountered but no detail } \\
\text { provided on how these data were } \\
\text { collected. }\end{array}$} \\
\hline \multirow[t]{5}{*}{ M-SPAN } & USA & $\begin{array}{l}\text { Changes to PE lesson context, } \\
\text { structure and teacher behaviour } \\
\text { to increase physical activity. }\end{array}$ & & \\
\hline & Target group: & Environment: & & \\
\hline & $11-14$ years & $\begin{array}{l}\text { Physical activity was promoted } \\
\text { throughout the school day (e.g. } \\
\text { during breaks and lunchtimes). } \\
\text { School policies to support } \\
\text { physical activity and healthy } \\
\text { eating implemented. Changes } \\
\text { made to the nutritional quality } \\
\text { of food offered in schools. } \\
\text { Student health committees set } \\
\text { up to implement monthly } \\
\text { health-related activities. }\end{array}$ & & \\
\hline & Duration: & Family/community: & & \\
\hline & 2 years & $\begin{array}{l}\text { Intervention was promoted to } \\
\text { parents via articles in the school } \\
\text { newsletter, posters and brochures } \\
\text { at open houses and presentations } \\
\text { to Parent Teacher Association } \\
\text { meetings. }\end{array}$ & & \\
\hline
\end{tabular}


Table 1 Characteristics of studies (Continued)

\begin{tabular}{|c|c|c|c|c|}
\hline $\begin{array}{l}\text { Trevino et al. } \\
2004[55,56]\end{array}$ & & & \multirow{6}{*}{$\begin{array}{l}\text { Fitness scores }(p=0.04) \text { and dietary } \\
\text { fibre intake }(p=0.09) \text { increased } \\
\text { significantly in intervention children } \\
\text { compared to controls. }\end{array}$} & \multirow{6}{*}{$\begin{array}{l}\text { Focus groups conducted to explore } \\
\text { barriers to family involvement. No details } \\
\text { provided for methods for assessing } \\
\text { fidelity/intensity. }\end{array}$} \\
\hline \multirow[t]{5}{*}{ Bienestar } & USA & $\begin{array}{l}50 \times 45 \text { minute health education } \\
\text { sessions throughout the intervention. } \\
\text { Curriculum focuses on nutrition, } \\
\text { physical activity, self-esteem, self- } \\
\text { control, and diabetes mellitus. }\end{array}$ & & \\
\hline & Target group: & Environment: & & \\
\hline & 9-10 years & $\begin{array}{l}\text { School food service staff receive } \\
\text { nutritional training. Bienestar } \\
\text { health club held once a week } \\
\text { after school. }\end{array}$ & & \\
\hline & Duration: & Family/community: & & \\
\hline & 5 months & $\begin{array}{l}\text { Variety of parent 'fun' activities are } \\
\text { held including: cooking } \\
\text { demonstrations, salsa classes and } \\
\text { games. Parent meetings. }\end{array}$ & & \\
\hline \multirow{6}{*}{$\begin{array}{l}\text { Williamson } \\
\text { et al. } 2012 \\
\text { Louisiana (LA) } \\
\text { HEALTH } \\
{[57,58]}\end{array}$} & Country: & Curriculum: & \multirow{6}{*}{$\begin{array}{l}\text { No differences between } \\
\text { intervention and control students } \\
\text { for body fat and BMI z-scores. }\end{array}$} & \multirow{6}{*}{$\begin{array}{l}\text { Questionnaires and observations used to } \\
\text { assess integrity of delivery (No further } \\
\text { details provided). Tracking system used to } \\
\text { monitor usage of internet component }\end{array}$} \\
\hline & USA & $\begin{array}{l}\text { Weekly classroom lessons ( } 20-25 \\
\text { mins) on healthy eating and exercise } \\
\text { implemented by teachers, as well as } \\
\text { additional internet lessons. }\end{array}$ & & \\
\hline & Target group: & Environment: & & \\
\hline & 9-12 years & $\begin{array}{l}\text { Health promotion campaigns } \\
\text { carried out in classrooms, } \\
\text { hallways and other locations } \\
\text { within the school. Modifications } \\
\text { to school food provision to } \\
\text { increase healthy options. Catering } \\
\text { staff training. Vending machines } \\
\text { provide healthy options. Regular } 5 \\
\text { minute activity breaks in } \\
\text { classrooms. Physical activity } \\
\text { equipment. }\end{array}$ & & \\
\hline & Duration: & Family/community: & & \\
\hline & 2.5 years & $\begin{array}{l}\text { Bi-monthly newsletters sent home } \\
\text { to parents. Family homework } \\
\text { assignments. Healthy menus sent } \\
\text { to parents. }\end{array}$ & & \\
\hline
\end{tabular}

Abbreviations used in table: BMI (Body Mass Index), zBMI (Body Mass Index, standardized by age and gender), MVPA (moderate-to-vigorous physical activity), $P E$ (Physical Education), $C I$ (confidence interval).

parents. Even in the $C A T C H$ study where $70 \%$ of parents participated in some intervention activities, the authors noted the intensity of the family component was very low and thus unable to produce significant changes in children's health behaviours [59].

A report on the Bienestar programme targeting Mexican Americans [55] identified very low family participation rates (17\%), and study investigators conducted focus groups with parents to explore why [56]. Explanations included: misunderstanding the purpose of the programme and the family events offered; practical issues such as lack of transportation, babysitting or limited income; time constraints and conflicts with work schedules; embarrassment concerning parents' own education and/or literacy levels; expectations of 'boring', didactic, teacher-led meetings; too many meetings offered; and assumptions that meetings were just for mothers and excluded other family members. In response, the program was modified to encourage greater participation leading to an increase in parental involvement, but nonetheless still only resulting in about a third of parents becoming involved (increase from $17 \%$ to $37 \%$ ).

\section{Facilitators of implementation}

Making interventions relevant to the specific school context was noted as important. Needs assessments were undertaken in schools in some studies [32,52] helping to create interest and motivation within schools. Several studies encouraged schools to tailor intervention components to local needs. Equally, materials and messages needed to be culturally relevant, especially when targeting specific ethnic groups [43,55], helping create local ownership. 
Good communication and thorough training were also noted as essential. Nicklas and O'Neill [29] explained that schools need to feel confident they are able to carry out the required tasks and adequate training is essential in achieving implementation fidelity. Researchers on the 5 A Day Power Plus study similarly recognised training and staff development as critical to implementation [31]. Teachers in this study received on-going training with one-on-one feedback and support. To facilitate this, the study reimbursed schools for the cost of substitute teachers and paid catering staff to attend training.

Understanding schools' 'core business' also appeared critical. As Nicklas and O'Neill pointed out, successful school health interventions' objectives aligned with teachers' goals for their students [29]. Radcliffe et al. [32], for example, explained that schools in their study saw the intervention programme as related to the core business of the school. A needs assessment identified lack of breakfast as a particular problem and teachers were convinced that this was associated with poorer concentration and classroom behaviour. Disappointingly this study did not go on to measure the intervention's impact on these educational outcomes.

Working with schools to develop programmes was similarly important. Teachers could help develop both the intervention programme and its delivery plan to ensure relevancy and increase implementation fidelity [36]. Participation from students could also be useful [26]. Brandstetter et al. [42] advocated the need for pragmatic programmes that fit within the existing curriculum and school structures without creating additional demands on teachers.

\section{Barriers to implementation}

Competing priorities and lack of institutional support were noted as barriers to successful implementation. Both the HEALTHY [48] and Pathways [45] studies cited the emphasis on academic subjects over PE as hindering implementation. One programme co-ordinator from the HEALTHY study noted that 'at times, the administration was pulling students out of PE to do some academic testing, and we had situations where students were working on writing assignments during PE because of pressures from administration'([48]:p313). Similarly, Story and colleagues suggested that implementation of the 5 A DAY Power Plus programme in the fifth grade dropped because media reports of low academic test scores within the district meant teachers refocused only on 'teaching the basics' [31]. The intervention programme was thus an additional pressure which could easily be dropped. Other studies suggested that preparation for a forthcoming educational inspection or a general lack of time compromised teachers' ability to engage in and deliver the intervention $[27,36,45,52]$.

Numerous practical issues also presented challenges to implementation including: lack of space to deliver PE lessons [45]; difficulties in delivering hands-on taste testing sessions [34]; teacher absences or rapid staff turnover [41,52]; high student-to-teacher ratios [48]; and lack of volunteers to run after-school physical activities [54]. Concerns over teacher burn-out and disruptive student behaviour were also mentioned $[45,48]$.

Some issues that affected intervention delivery and success were beyond schools' control. Fry and colleagues [41], for example, described how creating safe walking route to schools meant tackling local infrastructure, traffic management and access to public transport. Some school principals talked to local authorities about addressing these issues with varying success. Sallis et al. [54] identified the need for school food services to be financially self-supporting as the greatest barrier to improving student nutrition. Offering unfamiliar (and potentially unpopular) healthy foods posed too great a financial risk for catering services and thus disincentivised change. In addition, a centralized kitchen system meant schools had little control over ingredients or preparation.

Finally, although interventions must be of sufficient length and intensity to enable behavioural change and health impacts, optimal duration is not clear from these studies, there being no clear association between intervention duration and health impact.

\section{Discussion}

The HPS framework is generally effective at increasing physical activity, fitness and fruit and vegetable intake in school students [17]. This paper looked in more detail at the implementation of physical activity and/or nutrition interventions and identified key factors helping or hindering implementation and/or success.

\section{Summary of main findings}

Process evaluations revealed high levels of acceptability among teachers and students, but implementation fidelity varied considerably across trials. In particular, involving families, despite being a key part of the HPS approach, was reported as highly challenging. Essential elements of interventions included: tailoring programmes to individual schools' needs; aligning interventions with schools' core aims; working with teachers to develop programmes and increase ownership; and providing on-going training, support and communication. The emphasis on academic subjects (and the corresponding low value placed on health initiatives), and lack of institutional support were cited as barriers to implementation.

Many of these findings are congruent with conclusions from other studies examining effective elements of school-based interventions. A recent narrative synthesis of qualitative studies adopting the HPS approach [60] identified the importance of institutional support, assessment of school needs, ownership of programmes, adequate 
training and tailoring of intervention components to local contexts. This review also noted the low value placed on health versus academic achievement as a barrier to effective implementation. Similar findings regarding intervention development and implementation are also noted in Peters et al.'s recent review of school health promotion [61] and in guidelines for HPS produced by the International Union for Health Promotion and Education [14].

\section{Limitations}

Most studies lacked detailed description of intervention components and activities which would enable replication in other contexts. Equally, the quality of process data varied considerably and was often poorly reported. While providing some useful insights, much of the process data presented by these studies was disappointing in terms of its scope and depth. Process data generally consisted of quantitative assessments of acceptability and/or fidelity. While important, these are insufficient to explain why some interventions failed while others succeeded. For example, while studies which reported positive intervention impacts also reported high levels of implementation fidelity, so did many other studies which found no such positive effects. The most useful insights into intervention success often came from authors' reflections reported in the discussion sections of papers, despite the evidence for many such claims being unclear. However, it should be noted that findings reported here are often based on reports from just one or two studies.

Better designed, more comprehensive process evaluations that go beyond mere quantitative assessments of acceptability and/or fidelity are required. These should provide greater insight into the context in which interventions are implemented and how this can affect intervention success. It is also notable that almost a quarter of the 34 physical activity and/or nutrition interventions identified by the Cochrane review provided no process data and were thus excluded from this analysis.

The publication of the template for intervention description and replication (TIDieR) checklist and guide [62] and the recently published process evaluation guidance [63] from the UK's Medical Research Council are welcome developments. The adoption of these guidelines by scientific journals may prove important in raising both the profile and quality of reporting of trials and associated process evaluations. This is particularly important for multi-component, complex interventions where it is important to identify, first, what is being standardised in the intervention (intervention components or steps in the change process [64]) and, second, what works, for whom, in what circumstances and why [18].

That this study focuses only on RCT evidence is both a strength and a limitation. While evidence from RCTs provides the most reliable means of assessing intervention effectiveness, we acknowledge that many evaluations of the HPS approach do not use this methodology and thus were excluded from this review $[11,14,15]$. However, findings reported here are congruent with reviews of the wider evidence base $[14,16]$ and contribute to the emerging picture of how best to improve physical activity and nutrition in schools. Implementation trials (including process evaluations) that evaluate the roll-out of successful programmes would extend our understanding of how to implement such interventions in 'real world' settings.

\section{Implications for policy and research}

Our findings raise three challenges for both policy makers and researchers. First, we need greater integration between health and education [65]. As suggested by the findings described above, schools are more likely to engage in health interventions if they fit with institutional priorities, namely improving educational attainment. It is disappointing that none of these HPS interventions measured outcomes such as academic test scores, attendance, attention, concentration, behaviour in the classroom or attitude towards school. Overweight and obesity have been found to be associated with poor academic performance [66]. There is also some evidence to suggest physical activity $[67,68]$ and nutrition (particularly breakfast schemes) [68-71] can improve academic achievement. However, these data are often from methodologically weak studies and causality has yet to be demonstrated [72]. It is imperative that rigorous RCT evaluation studies include both health and educational outcomes to determine effectiveness, thus speaking to policy makers in health and education sectors alike.

Second, school health researchers need to carefully consider the importance of family involvement within HPS interventions. Evidence for the impact of family involvement in school-based obesity interventions remains inconclusive; some reviews suggest its importance $[7,73]$ while others report no consistent pattern [74,75]. It may be that this aspect of the HPS framework is impractical in some or all schools and interventions would do better to focus resources on 'in-school' activities. Or it may simply be, as this review has found, that current approaches to parental involvement are inadequate (for example, newsletters or information evenings) and more innovative methods are required. Further research - both qualitative and quantitative - is needed to address these questions.

Finally, we need more evaluations (including process evaluations that go beyond quantitative measures of fidelity and acceptability) of interventions to promote physical activity and nutrition during adolescence. Physical activity levels are known to decline during teenage years, particularly in young women [76,77]. Adolescence 
also represents a period when young people start to make their own choices over the food they eat and how they spend their time [78]. The lack of research into this critical age period is therefore disappointing and represents a missed opportunity for public health impact.

\section{Conclusion}

The HPS framework has been shown to be effective overall in improving physical activity and nutrition, the key determinants of overweight and obesity [17]. The process data reported in these trials offer important insights into essential elements of success, as well as the challenges to implementation which need to be addressed at the outset of any new programme. These data suggest that the success of the HPS approach lies in creating effective partnerships between researchers, schools and families.

\section{Competing interests}

The authors declare that they have no competing interests.

\section{Authors' contributions}

$\mathrm{RL}$ performed the searches, extracted and analysed the process data and wrote the first draft of the manuscript. RC, HJ and CB extracted data, provided critical commentary on this paper and helped draft the final manuscript. All authors read and approved the final manuscript.

\section{Acknowledgements}

We are grateful to our co-authors on the original Cochrane systematic review: Theodora Pouliou, Simon Murphy, Elizabeth Waters, Kelli Komro, Lisa Gibbs and Daniel Magnus. We also gratefully acknowledge the support of Geraldine Macdonald and all at the Cochrane Developmental, Psychosocial and Learning Problems Group.

The work was undertaken with the support of The Centre for the Development and Evaluation of Complex Interventions for Public Health Improvement (DECIPHer), a UKCRC Public Health Research Centre of Excellence. Joint funding (MR/KO232331/1) from the British Heart Foundation, Cancer Research UK, Economic and Social Research Council, Medical Research Council, the Welsh Government and the Wellcome Trust, under the auspices of the UK Clinical Research Collaboration, is gratefully acknowledged. The funders had no role in the study design, data extraction, data analysis, data interpretation, or writing of the report.

\section{Author details}

'DECIPHer, School of Social and Community Medicine, University of Bristol, Canynge Hall, 39 Whatley Rd, Bristol BS8 2PS, UK. ${ }^{2}$ Social Science Research Unit, Institute of Education, University College London, 20 Bedford Way, London WC1H OAL, UK. ${ }^{3}$ School of Social and Community Medicine, University of Bristol, Canynge Hall, 39 Whatley Rd, Bristol BS8 2PS, UK.

Received: 11 June 2014 Accepted: 13 January 2015

Published online: 13 February 2015

\section{References}

1. Wang $Y$, Lobstein T. Worldwide trends in childhood overweight and obesity. Int J Pediatr Obes. 2006;1(1):11-25.

2. Ogden CL, Carroll MD, Kit BK, Flegal KM. Prevalence of obesity and trends in body mass index among US children and adolescents, 1999-2010. JAMA. 2012;307(5):483-90.

3. Wang $Y$, Lim H. The global childhood obesity epidemic and the association between socio-economic status and childhood obesity. Int rev psychiatry. 2012;24(3):176-88.

4. Singh AS, Mulder C, Twisk JWR, Van Mechelen W, Chinapaw MJM. Tracking of childhood overweight into adulthood: a systematic review of the literature. Obes Rev. 2008;9(5):474-88.
5. Reilly J, Kelly J. Long-term impact of overweight and obesity in childhood and adolescence on morbidity and premature mortality in adulthood: systematic review. Int J Obes. 2011;35:891-8.

6. World Health Orgzaniation. Population-based approaches to childhood obesity prevention. Geneva: World Health Organization; 2012.

7. Waters E, de Silva-Sanigorski A, Hall B, Brown T, Campbell K, Gao Y, et al. Interventions for preventing obesity in children. Cochrane Database of Systematic Reviews. 2011 (12):Art. No.: CD001871. doi:10.1002/14651858. CD001871.pub3.

8. World Health Orgzaniation. Promoting health through schools. Report of a WHO expert committee on comprehensive school health education and promotion, WHO Technical Report Series 870. Geneva: World Health Organization; 1997.

9. World Health Organization. Health Promoting Schools: A healthy setting for living, learning, working. Geneva: World Health Organization; 1998.

10. Young I, Williams T. The Healthy School. Scottish Health Education Group: Edinburgh; 1989

11. Lister-Sharp D, Chapman S, Stewart-Brown S, Sowden A. Health promoting schools and health promotion in schools: two systematic reviews. Health Technol Assess. 1999;3(22):1-209.

12. Mũkoma W, Flisher AJ. Evaluations of health promoting schools: a review of nine studies. Health Promot Int. 2004;19(3):357-68.

13. Lynagh M, Schofield MJ, Sanson-Fisher RW. School Health Promotion Programs Over the Past Decade: A Review of the Smoking, Alcohol and Solar Protection Literature. Health Promot Int. 1997;12(1):43-60.

14. IUHPE. Achieving health promoting schools: guidelines for promoting health in schools. Version 2 of the document formerly known as "Protocols and guidelines for health promoting schools". International Union for Health Promotion and Education 2008. Available from http://www.dhhs.tas. gov.au/_data/assets/pdf_file/0011/115895/guidelines_for_health_promoting_ schools1.pdf, accessed 30 Oct 2014.

15. Stewart-Brown S. What is the evidence on school health promotion in improving or preventing disease and, specifically, what is the effectiveness of the health promoting schools approach? Cophenhagen: WHO Regional Office for Europe (Health Evidence Network report: http://www.euro.who. int/_data/assets/pdf_file/0007/74653/E88185.pdf, accessed 30 Oct 2014), 2006.

16. IUHPE. Promoting Health in Schools: From Evidence to Action. International Union for Health Promotion and Education. Available from http://www.iuhpe. org/images/PUBLICATIONS/THEMATIC/HPS/Evidence-Action_ENG.pdf, accessed 30 Oct 2014, 2010.

17. Langford R, Bonell C, Jones H, Pouliou T, Murphy S, Waters E, et al. The WHO Health Promoting School framework for improving the health and well-being of students and their academic achievement. Cochrane Database of Systematic Reviews. 2014;Issue 4. Art. No.: CD008958. doi:10.1002/ 14651858.CD008958.pub2.

18. Pawson R, Tilley N. Realistic evaluation. London: Sage; 1997.

19. Thomas J, Harden A. Methods for the thematic synthesis of qualitative research in systematic reviews. BMC Med Res Methodol. 2008;8(1):45.

20. Pope C, Ziebland S, Mays N. Qualitative research in health care. BMJ. 2000;320(7227):114-6.

21. Gale N, Heath G, Cameron E, Rashid S, Redwood S. Using the framework method for the analysis of qualitative data in multi-disciplinary health research. BMC Med Res Methodol. 2013;13(1):117.

22. Bere $\mathrm{E}$, Veierod MB, Bjelland M, Klepp Kl. Outcome and process evaluation of a Norwegian school-randomized fruit and vegetable intervention: Fruits and Vegetables Make the Marks (FVMM). Health Educ Res. 2006;21(2):258-67.

23. Evans CE, Ransley JK, Christian MS, Greenwood DC, Thomas JD, Cade JE. A cluster-randomised controlled trial of a school-based fruit and vegetable intervention: Project Tomato. Public Health Nutr. 2013;16(6):1073-81.

24. Foster GD, Sherman S, Borradaile KE, Grundy KM, Vander Veur SS, Nachmani J, et al. A Policy-Based School Intervention to Prevent Overweight and Obesity. Pediatrics. 2008;121(4):e794-802.

25. Hoffman JA, Franko DL, Thompson DR, Power TJ, Stallings VA. Longitudinal behavioral effects of a school-based fruit and vegetable promotion program. J Pediatr Psychol. 2010;35(1):61-71.

26. Hoppu U, Lehtisalo J, Kujala J, Keso T, Garam S, Tapanainen H, et al. The diet of adolescents can be improved by school intervention. Public Health Nutr. 2010;13(6A):973-9.

27. Lytle LA, Murray DM, Perry CL, Story M, Birnbaum AS, Kubik MY, et al. School-based approaches to affect adolescents' diets: results from the TEENS study. Health Educ Behav. 2004;31(2):270-87. 
28. Nicklas TA, Johnson CC, Myers L, Farris RP, Cunningham A. Outcomes of a high school program to increase fruit and vegetable consumption: Gimme 5-A fresh nutrition concept for students. J Sch Health. 1998;68(6):248-53.

29. Nicklas TA, O'Neil CE. Process of Conducting a 5-a-Day Intervention with High School Students: Gimme 5 (Louisiana). Health Educ Behav. 2000;27 (2):201-12.

30. Perry CL, Bishop DB, Taylor G, Murray DM, Mays RW, Dudovitz BS, et al. Changing fruit and vegetable consumption among children: the 5-a-day power plus program in St. Paul, Minnesota. Am J Public Health. 1998;88 (4):603-9.

31. Story M, Mays RW, Bishop DB, Perry CL, Taylor G, Smyth M, et al. 5-a-Day Power Plus: process Evaluation of a Multicomponent Elementary School Program to Increase Fruit and Vegetable Consumption. Health Educ Behav. 2000;27(2):187-200.

32. Radcliffe B, Ogden C, Welsh J, Carroll S, Coyne T, Craig P. The Queensland School Breakfast Project: a health promoting schools approach. Nutr Diet. 2005;62:33-40.

33. Reynolds KD, Franklin FA, Binkley D, Raczynski JM, Harrington KF, Kirk KA, et al. Increasing the fruit and vegetable consumption of fourth-graders: results from the High 5 project. Prev Med. 2000;30(4):309-19.

34. Reynolds KD, Franklin FA, Leviton LC, Maloy J, Harrington KF, Yaroch AL, et al. Methods, results, and lessons learned from process evaluation of the high 5 school-based nutrition intervention. Health Educ Behav. 2000;27(2):177-86.

35. te Velde SJ, Brug J, Wind M, Hildonen C, Bjelland M, Perez-Rodrigo C, et al. Effects of a comprehensive fruit- and vegetable-promoting school-based intervention in three European countries: the Pro Children Study. Br J Nutr. 2008:99(4):893-903.

36. Wind M, Bjelland M, Pérez-Rodrigo C, Tevelde SJ, Hildonen C, Bere E, et al. Appreciation and implementation of a school-based intervention are associated with changes in fruit and vegetable intake in 10- to 13-year old schoolchildren The Pro Children study. Health Educ Res. 2008;23(6):997-1007.

37. Eather N, Morgan P, Lubans D. Improving the fitness and physical activity levels of primary school children: results of the Fit-4-Fun randomized controlled trial. Prev Med. 2013;56:12-9.

38. Kriemler S, Zahner L, Schindler C, Meyer U, Hartmann T, Hebestreit H, et al. Effect of school based physical activity programme (KISS) on fitness and adiposity in primary schoolchildren: cluster randomised controlled trial. BMJ. 2010;340:C785.

39. Simon C, Wagner A, Platat C, Arveiler D, Schweitzer B, Schlienger $J$, et al. ICAPS: a multilevel program to improve physical activity in adolescents. Diabetes Metab. 2006;32(1):41-9.

40. Wen LM, Fry D, Merom D, Rissel C, Dirkis H, Balafas A. Increasing active travel to school: are we on the right track? A cluster randomised controlled trial from Sydney. Prev Med. 2008;47(6):612-8.

41. Fry D, Wen L, Merom D, Dirkis H, Rissel C, Balafas A. The Central Sydney Walk to School research program 2005-2007. New South Wales Department of Health: Sydney; 2009.

42. Brandstetter S, Klenk J, Berg S, Galm C, Fritz M, Peter R, et al. Overweight prevention implemented by primary school teachers: a randomised controlled trial. Obes Facts. 2012;5:1-11.

43. Caballero B, Clay T, Davis SM, Ethelbah B, Rock BH, Lohman T, et al. Pathways: a school-based, randomized controlled trial for the prevention of obesity in American Indian schoolchildren. Am J Clin Nutr. 2003;78(5):1030-8.

44. Davis SM, Clay T, Smyth M, Gittelsohn J, Arviso V, Flint-Wagner H, et al. Pathways curriculum and family interventions to promote healthful eating and physical activity in American Indian schoolchildren. Prev Med. 2003;37((6 part 2):S24-34.

45. Gittelsohn J, Merkle S, Story M, Stone EJ, Steckler A, Noel J, et al. School climate and implementation of the Pathways study. Prev Med. 2003;37(6 part 2):S97-106.

46. Crespo NC, Elder JP, Ayala GX, Slymen DJ, Campbell NR, Sallis JF, et al. Results of a multi-level intervention to prevent and control childhood obesity among Latino children: the Aventuras Para Ninos study. Ann Behav Med. 2012;43:84-100.

47. Foster GD, Linder B, Baranowski T, Cooper DM, Goldberg L, Harrell JS, et al. A school-based intervention for diabetes risk reduction. N Engl J Med. 2010;363(5):443-53.

48. Hall W, Zeveloff A, Steckler A, Schneider M, Thompson D, Pham T, et al. Process evaluation results from the HEALTHY physical education intervention. Health Educ Res. 2012;27(2):307-18.

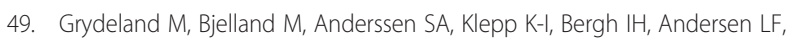
et al. Effects of a 20-month cluster randomised controlled school-based intervention trial on BMl of school-aged boys and girls: the HEIA study. Br J Sports Med. 2013. doi:10.1136/bjsports-2013-092284.

50. Haerens L, Deforche B, Maes L, Stevens V, Cardon G, De Bourdeaudhuij I. Body mass effects of a physical activity and healthy food intervention in middle schools. Obesity. 2006;14(5):847-54.

51. Luepker RV, Perry CL, McKinlay SM, Nader PR, Parcel GS, Stone EJ, et al. Outcomes of a field trial to improve children's dietary patterns and physical activity. The Child and Adolescent Trial for Cardiovascular Health (CATCH). JAMA. 1996;275(10):768-76.

52. Sahota P, Rudolf MCJ, Dixey R, Hill AJ, Barth JH, Cade J. Randomised controlled trial of primary school based intervention to reduce risk factors for obesity. BMJ. 2001;323(7320):1029-32.

53. Sahota P, Rudolf MCJ, Dixey R, Hill AJ, Barth JH, Cade J. Evaluation of implementation and effect of primary school based intervention to reduce risk factors for obesity. BMJ. 2001;323(7320):1027-9.

54. Sallis JF, McKenzie TL, Conway TL, Elder JP, Prochaska JJ, Brown M, et al. Environmental interventions for eating and physical activity: a randomized controlled trial in middle schools. Am J Prev Med. 2003;24(3):209-17.

55. Trevino RP, Yin Z, Hernandez A, Hale DE, Garcia OA, Mobley C. Impact of the Bienestar school-based diabetes mellitus prevention program on fasting capillary glucose levels: a randomized controlled trial. Arch Pediatr Adolesc Med. 2004;158(9):911-7.

56. Garcia-Dominic O, Wray LA, Trevino RP, Hernandez AE, Yin Z, Ulbrecht JS. Identifying barriers that hinder onsite parental involvement in a schoolbased health promotion program. Health Promot Pract. 2010;11(5):703-13.

57. Gabriele J, Stewart T, Sample A, Davis A, Allen R, Martin C, et al. Development of an internet-based obesity prevention program for children. J Diabetes Sci Technol. 2010;4(3):723-32.

58. Williamson DA, Champagne CM, Harsha DW, Han H, Martin CK, Newton RL, et al. Effect of an environmental school-based obesity prevention program on changes in body fat and body weight: a randomized trial. Obesity. 2012;20:1653-61.

59. Nader PR, Sellers DE, Johnson CC, Perry CL, Stone EJ, Cook KC, et al. The effect of adult participation in a school-based family intervention to improve children's diet and physical activity: The child and adolescent trial for cardiovascular health. Prev Med. 1996;25(4):455-64.

60. Hung T, Chiang V, Dawson A, Lee R. Understanding of Factors that Enable Health Promoters in Implementing Health-Promoting Schools: a Systematic Review and Narrative Synthesis of Qualitative Evidence. PLoS One. 2014;9(9):e108284.

61. Peters L, Kok G, Ten Dam G, Buijs G, Paulussen T. Effective elements of school health promotion across behavioral domains: a systematic review of reviews. BMC Public Health. 2009;9(1):182.

62. Hoffmann TC, Glasziou PP, Boutron I, Milne R, Perera R, Moher D, et al. Better reporting of interventions: template for intervention description and replication (TIDieR) checklist and guide. BMJ. 2014;348:g1687.

63. Moore G, Audrey S, Barker M, Bond L, Bonell C, Hardeman W, et al. Process evaluation of complex interventions. UK Medical Research Council (MRC) guidance. London: MRC Population Health Science Research Network; 2014.

64. Hawe P, Shiell A, Riley T. Complex interventions: how "out of control" can a randomised controlled trial be? BMJ. 2004;328(7455):1561-3.

65. Mohammadi NK, Rowling L, Nutbeam D. Acknowledging educational perspectives on health promoting schools. Health Educ. 2010;110(4):240-51.

66. Taras H, Potts-Datema W. Obesity and Student Performance at School. J Sch Health. 2005;75(8):291-5.

67. Trudeau F, Shephard R. Physical education, school physical activity, school sports and academic performance. Int J Behav Nutr Phys Act. 2008;5(1):10.

68. Murray NG, Low BJ, Hollis C, Cross AW, Davis SM. Coordinated School Health Programs and Academic Achievement: a Systematic Review of the Literature. J Sch Health. 2007;77(9):589-600.

69. Cueto S. Breakfast and performance. Public Health Nutr. 2001;4(6a):1429-31.

70. Rasberry CN, Lee SM, Robin L, Laris BA, Russell LA, Coyle KK, et al. The association between school-based physical activity, including physical education, and academic performance: A systematic review of the literature. Prev Med. 2011;52(supplement):S10-20.

71. Hoyland A, Dye L, Lawton CL. A systematic review of the effect of breakfast on the cognitive performance of children and adolescents. Nutr Res Rev. 2009;22(02):220-43. 
72. Suhrcke M, de Paz NC. The impact of health and health behaviours on educational outcomes in high-income countries: a review of the evidence. WHO Regional Office for Europe: Copenhagen; 2011.

73. Nixon CA, Moore HJ, Douthwaite W, Gibson EL, Vogele C, Kreichauf S, et al. Identifying effective behavioural models and behaviour change strategies underpinning preschool- and school-based obesity prevention interventions aimed at 4-6-year-olds: a systematic review. Obes Rev. 2012;13:106-17.

74. Brown T, Summerbell C. Systematic review of school-based interventions that focus on changing dietary intake and physical activity levels to prevent childhood obesity: an update to the obesity guidance produced by the National Institute for Health and Clinical Excellence. Obes Rev. 2009;10(1):110-41.

75. Kriemler S, Meyer U, Martin E, van Sluijs EM, Andersen LB, Martin BW. Effect of school-based interventions on physical activity and fitness in children and adolescents: a review of reviews and systematic update. $\mathrm{Br} J$ Sports Med. 2011:45(11):923-30.

76. Allison KR, Adlaf EM, Dwyer JJ, Lysy DC, Irving HM. The decline in physical activity among adolescent students: a cross-national comparison. Can J Public Health. 2007;98(2):97-100.

77. Nader PR, Bradley RH, Houts RM, McRitchie SL, O'Brien M. Moderate-to-vigorous physical activity from ages 9 to 15 years. JAMA. 2008;300(3):295-305.

78. Fitzgerald A, Heary C, Nixon E, Kelly C. Factors influencing the food choices of Irish children and adolescents: a qualitative investigation. Health Promot Int. 2010;25(3):289-98.

\section{Submit your next manuscript to BioMed Central and take full advantage of:}

- Convenient online submission

- Thorough peer review

- No space constraints or color figure charges

- Immediate publication on acceptance

- Inclusion in PubMed, CAS, Scopus and Google Scholar

- Research which is freely available for redistribution 